\title{
Thalamic Afferents and Neocortical Arealization: An Ongoing Journey
}

\author{
Laura R. Fenlon and Rodrigo Suárez \\ Queensland Brain Institute, The University of Queensland, St Lucia, QLD, 4072, Australia \\ Review of Vue et al.
}

The neocortex is a functionally intricate structure that processes sensory, motor, and associative functions in a spatially organized manner. Different areas of the cortex become specified during development by intrinsic and extrinsic influences. A molecular map of cortical areas is present from early stages within the ventricular zones, in the form of graded expression of transcription factors within neural progenitors. This map becomes amplified by columnar proliferation and is later refined by thalamic input (Rakic, 1988; for review, see Alfano and Studer, 2013). However, numerous studies have shown that sensory disruption (e.g., whisker cauterization or eye enucleation) greatly modifies the arealization, cytoarchitecture, fine morphology, and electrical properties of the cortex (for examples, see Kahn and Krubitzer, 2002 and references therein). Similarly, changes in thalamocortical axon (TCA) innervation can exert profound influences on cortical identity. Ablation of the thalamus at birth results in gross effects on cortical cytoarchitecture (Wise and Jones, 1978). Moreover, portions of embryonic visual cortex transplanted into the somatosensory cortex of

Received July 4, 2013; revised July 29, 2013; accepted July 29, 2013.

We thank Linda J. Richards; Ilan Gobius; and the editors of The Journal of Neuroscience, Teresa Esch and John Maunsell, for comments on this manuscript.

Correspondence should be addressed to Laura R. Fenlon, Queensland Brain Institute, The University of Queensland, St Lucia, QLD, 4072, Australia.E-mail: l.fenlon@uq.edu.au.

DOI:10.1523/JNEUROSCI.2859-13.2013

Copyright $\odot 2013$ the authors $\quad 0270-6474 / 13 / 3313938-02 \$ 15.00 / 0$ neonatal rats can acquire characteristics of the host tissue, including organization into barrels and innervation by somatosensory TCAs (Schlaggar and O'Leary, 1991). In contrast, genetic alteration of the presence or position of TCAs at embryonic stages has resulted in negligible effects in size, position, and molecular identity of cortical areas (Nakagawa et al., 1999; Garel et al., 2002). However, the genetic strategy used to alter TCAs in those studies also disrupted other regions crucial for normal development and the animals died before a postnatal analysis of cortical areas could be performed. Thus, the role of TCAs in the establishment of cortical maps has been uncertain.

Recently, Vue et al. (2013) investigated the role of TCAs in cortical arealization using three mouse lines with genetic alterations restricted to the thalamus and that were able to survive into adulthood: one with over-exuberant visual TCA projections from the dorsal lateral geniculate nucleus (dLGN), a second with reduced visual TCA projections, and a third with a nearly complete absence of TCAs. Examination of embryonic animals in these three lines revealed no observable difference in cortical arealization, confirming previous findings (Nakagawa et al., 1999; Garel et al., 2002). However, a battery of cortical areal markers at postnatal day (P) 8 revealed that increasing or reducing visual TCAs resulted in a corresponding increase or decrease in the size of the primary visual cortex (V1). This expansion or retraction of molecular areas de- marcating V1 was at the expense or gain of surrounding cortical areas that are usually responsible for higher-order cortical processing, although whether these areas are reduced/expanded or spatially shifted is not completely clear. Finally, in mice in which almost all TCAs were genetically eliminated, cortical areal boundaries became indistinct, providing further evidence that TCAs are necessary for normal segregation of cortical areas (Vue et al., 2013, their Fig. 10).

Another study, published shortly after that of Vue et al. (2013), genetically ablated nearly all visual TCAs from the dLGN and investigated the resulting cortical arealization at P7. Specifically, Chou et al. (2013) investigated the role of postnatal thalamic input in the distinction between V1 and higher-order visual areas. Similar to the findings of Vue et al. (2013), Chou et al. (2013) found that after visual TCA ablation, molecular markers that usually demarcate V1 from surrounding higher-order visual areas, were homogenously expressed across the entire visual cortex. This finding adds to the impact of Vue et al.'s (2013) experiments, further demonstrating that ablation of thalamocortical afferents to the sensory cortices results in a loss of spatial confinement of molecular markers specific for those cortical areas. The studies' conclusions are further strengthened by the differing techniques of the two articles: Chou et al. (2013) genetically ablated TCAs specifically from the dLGN, whereas Vue et al. (2013) performed a thalamus-wide abla- 
tion with similar results. This indicates that the observed cortical homogeneity upon both manipulations results from the absence of TCAs and is not a function of the specificity or generality of ablation. Finally, in the conditional knock-out animal used by Chou et al. (2013), TCAs developed normally until birth, and were then almost completely eliminated during early postnatal stages, whereas the mice used by Vue et al. (2013) show deficits in TCA targeting from embryonic stages. Thus, Chou et al. (2013) demonstrate that postnatal thalamic disruptions are sufficient to induce cortical area homogeneity and subsequently reinforce the hypothesis that TCAs only begin to play a crucial role in arealization after birth. Together, the findings of these two papers complement one another and significantly add to the body of literature examining the complex relationship between the thalamus and the cortex.

In conclusion, the findings of Vue et al. (2013) bridge the gap between classic studies showing that sensory input affects the development of postnatal cortical areas, and more recent genetic studies showing that embryonic cortical arealization is not affected by TCAs. By following the effects of thalamic size manipulations throughout cortical development, the authors demonstrate that cortical arealization is a multistep process. Cortical areas are predefined at embryonic stages, but they become refined postnatally after innervation of TCAs and sensory experience conveyed through them.

Although recent studies of cortical arealization have focused on molecular markers, this approach raises the questions: what features best define a cortical area? Is the connectivity and functionality of the redefined domains [e.g., the expanded V1 produced by Vue et al. (2013)] consistent with their new molecular profiles? Assimacopoulos et al. (2012) have recently induced functional duplication of cortical areas by overexpressing morphogens in early cortical progenitors. It would be interesting to investigate whether specific manipulations of the thalamus also result in functional changes of the cortical areas affected.

The precise mechanisms by which TCAs affect cortical organization remain open for further investigation. Future research combining examination of electrical activity, gene expression, and circuit formation will allow a better understanding of the relative roles of intrinsic and extrinsic factors in the development of the brain.

\section{References}

Alfano C, Studer M (2013) Neocortical arealization: evolution, mechanisms and open questions. Dev Neurobiol 73:411-447. CrossRef Medline

Assimacopoulos S, Kao T, Issa NP, Grove EA (2012) Fibroblast growth factor 8 organizes the neocortical area map and regulates sensory map topography. J Neurosci 32:71917201. CrossRef Medline

Chou SJ, Babot Z, Leingärtner A, Studer M, Nakagawa Y, O'Leary DD (2013) Geniculocortical input drives genetic distinctions between primary and higher-order visual areas. Science 340:1239-1242. CrossRef Medline

Garel S, Yun K, Grosschedl R, Rubenstein JL (2002) The early topography of thalamocortical projections is shifted in Ebf1 and Dlx1/2 mutant mice. Development 129: 5621-5634. CrossRef Medline

Kahn DM, Krubitzer L (2002) Massive crossmodal cortical plasticity and the emergence of a new cortical area in developmentally blind mammals. Proc Natl Acad Sci U S A 99: 11429-11434. CrossRef Medline

Nakagawa Y, Johnson JE, O'Leary DD (1999) Graded and areal expression patterns of regulatory genes and cadherins in embryonic neocortex independent of thalamocortical input. J Neurosci 19:10877-10885. Medline

Rakic P (1988) Specification of cerebral cortical areas. Science 241:170-176. CrossRef Medline

Schlaggar BL, O’Leary DD (1991) Potential of visual cortex to develop an array of functional units unique to somatosensory cortex. Science 252:1556-1560. CrossRef Medline

Vue TY, Lee M, Tan YE, Werkhoven Z, Wang L, Nakagawa Y (2013) Thalamic control of neocortical area formation in mice. J Neurosci 33:8442-8453. CrossRef Medline

Wise SP, Jones EG (1978) Developmental studies of thalamocortical and commissural connections in the rat somatic sensory cortex. J Comp Neurol 178:187-208. CrossRef Medline 